\title{
Design and Evaluation of a Sketch-Based Gesture Animation Tool
}

Huaguang Song and Michael Neff

University of California, Davis

\section{Abstract}

We present a sketch-based animation tool that allows non-expert users to efficiently create simple 3D animations of gesturing characters. Such animations are useful both to tell conversational stories and to develop gesture libraries for autonomous character systems. The tool operates by inviting the user to sketch paths on $2 \mathrm{D}$ planes that specify the $3 \mathrm{D}$ animation trace of the wrist, the variation of hand shape over time and to control wrist flexion. The goal of our system is to remove the complexity barrier of commercial animation packages in order to allow non-expert barrier of commercial animation packages in order to allow non-expert
users to create a variety of animations quickly. Controlling depth information and synchronizing various aspects of the animation are major challenges in using 2D sketch-based systems to produce 3D animation. The system provides two mechanisms for both depth and synchronization control. These are compared experimentally to better understand how a user can most effectively employ sketch to these tasks. Our results shown that all four methods are effective, but the use of synchronization markers makes this task easier than direct timing performance. Users were also quite satisfied with the overall output they generated with the system.

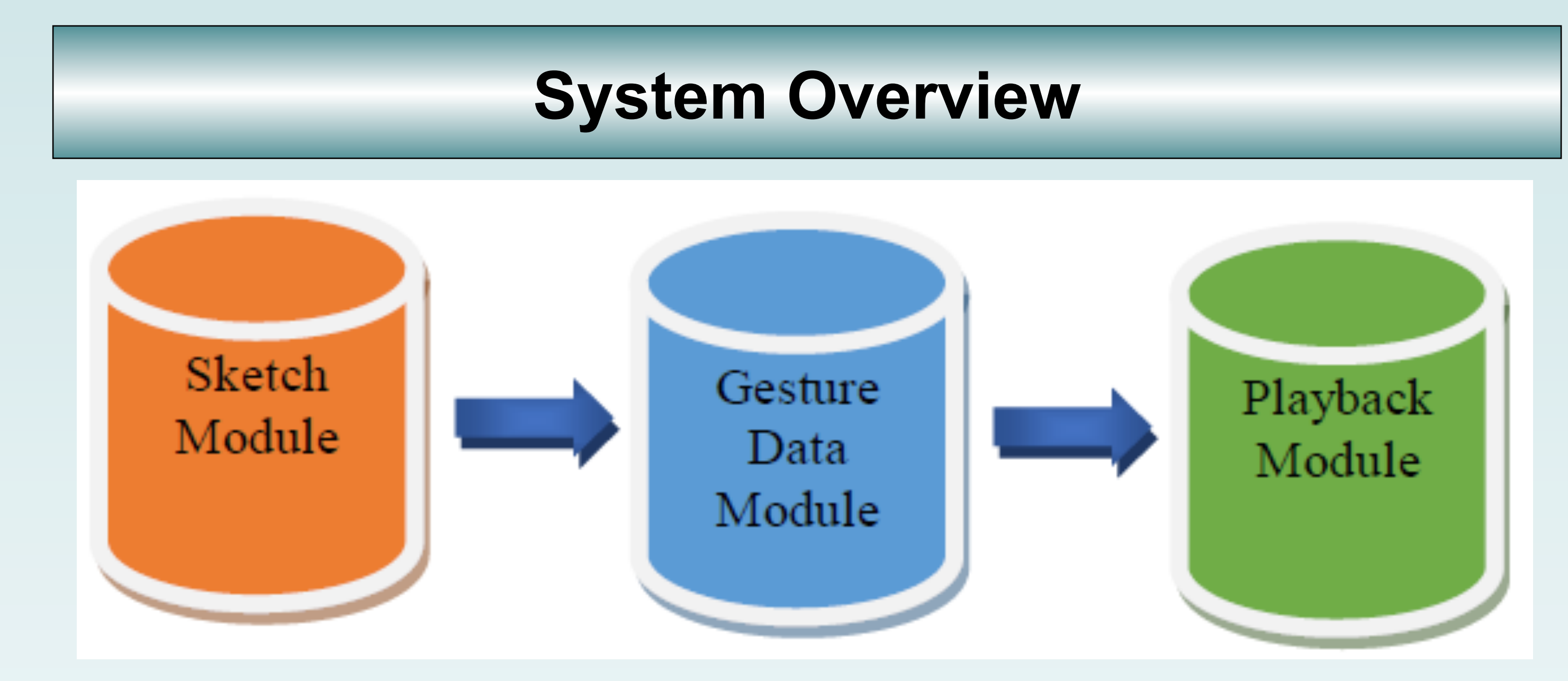

Figure 1: System Architecture

- Our system provides an intuitive drawing environment for novice users to quickly create gesture animation.

- We compare different methods for specifying depth information and synchronization across different channels of the animation. Experiments reveal that both alternatives are effective, but the use of alignment marks makes synchronization easier than direct timing control.

- Users were generally satisfied with the quality of the animation they could produce with the tool.

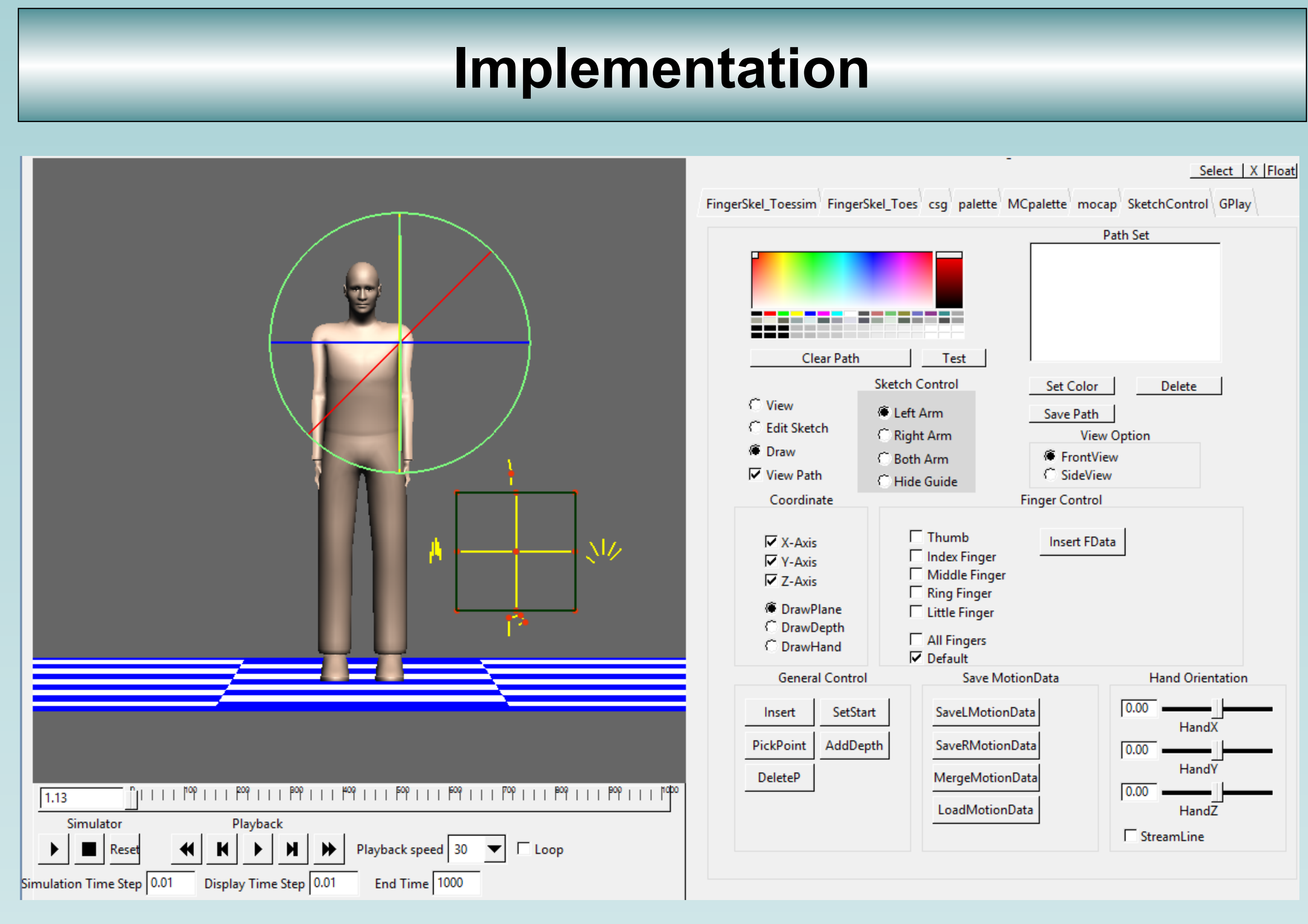

Figure 2: The main user interface consists of a sketch and display window on the left and a control palette on the right.
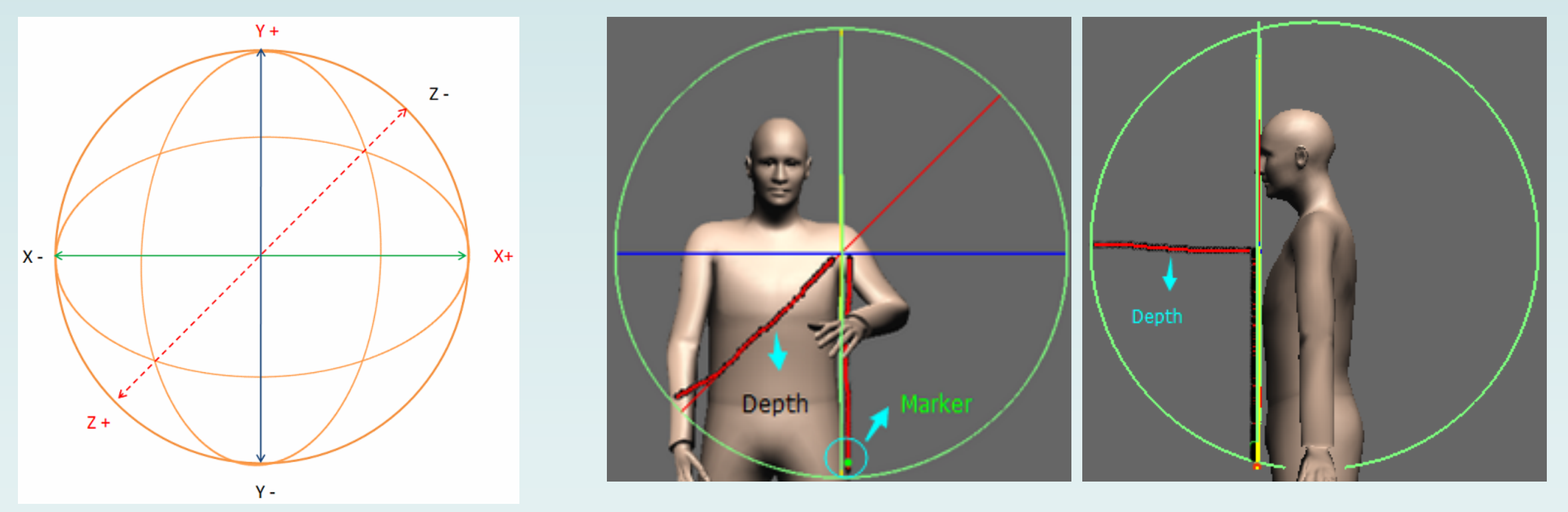

Figure 3: Coordinate System

Figure 4: Front and Side view with Depth Information
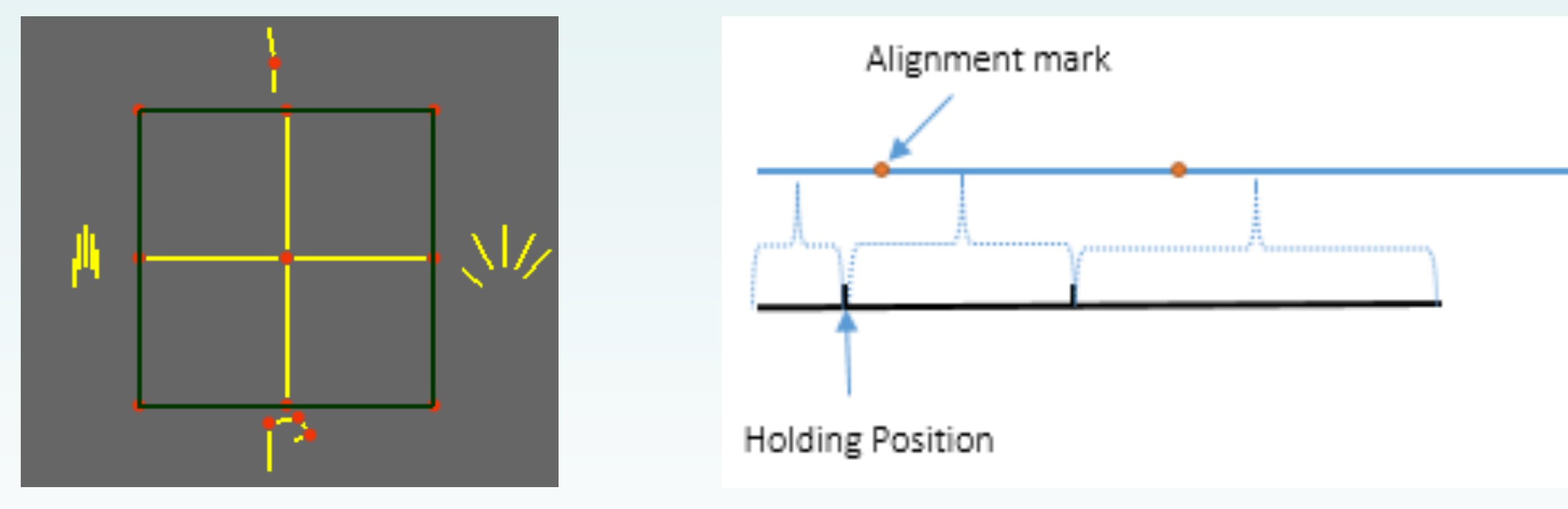

Figure 5: Input space for hand shapes Figure 6: Synchronization with Alignment marks

\section{Results}

Twenty subjects ( 8 male and 12 female) have been randomly chosen from the University community to participate in this study.

Each participant watched 9 short video clips of a person performing a gesture motion and then were asked to replicate the motion in animation using our sketch-based system

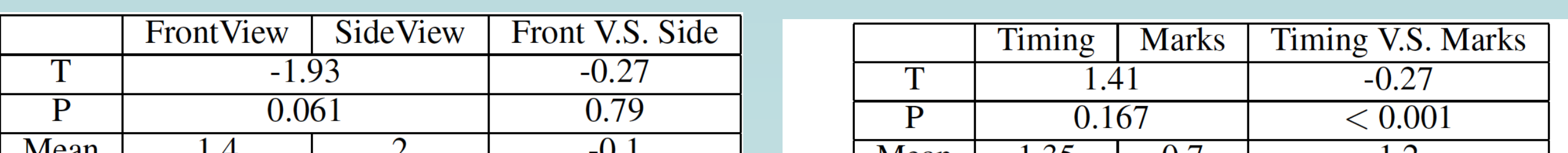

\begin{tabular}{|c|c|c|c|c|c|}
\hline $\mathrm{P}$ & \multicolumn{2}{|c|}{0.061} & 0.79 \\
\hline Mean & 1.4 & 2 & -0.1 \\
\hline
\end{tabular}$\quad$\begin{tabular}{|c|c|c|c|}
\hline P & \multicolumn{2}{|c|}{0.167} & $<0.001$ \\
\hline Mean & 1.35 & 0.7 & 1.2 \\
\hline
\end{tabular}

Table 1: Mean and T-test Results for Depth Control Table 2: Mean and T-test Results for Synchronization Control The subjects did not show a significant preference for either interface

\begin{tabular}{|l|c|c|c|c|}
\hline Tasks & \multicolumn{2}{|c|}{ Mean } & $\mathrm{T}$ & $\mathrm{P}$ \\
\hline 1a-1c & $\mathrm{F}: 3.5667$ & $\mathrm{~S}: 3.9667$ & -1.69 & 0.0996 \\
\hline 2a-2c & $\mathrm{T}: 3.5833$ & $\mathrm{M}: 3.9833$ & -3.35 & $<0.05$ \\
\hline
\end{tabular}

Table 4: Mean and t-test Score for Depth and Synchronization Control

Table 4 show that depth control using the front view $(F)$ or side view $(S)$ method does not show a significant difference for motion quality

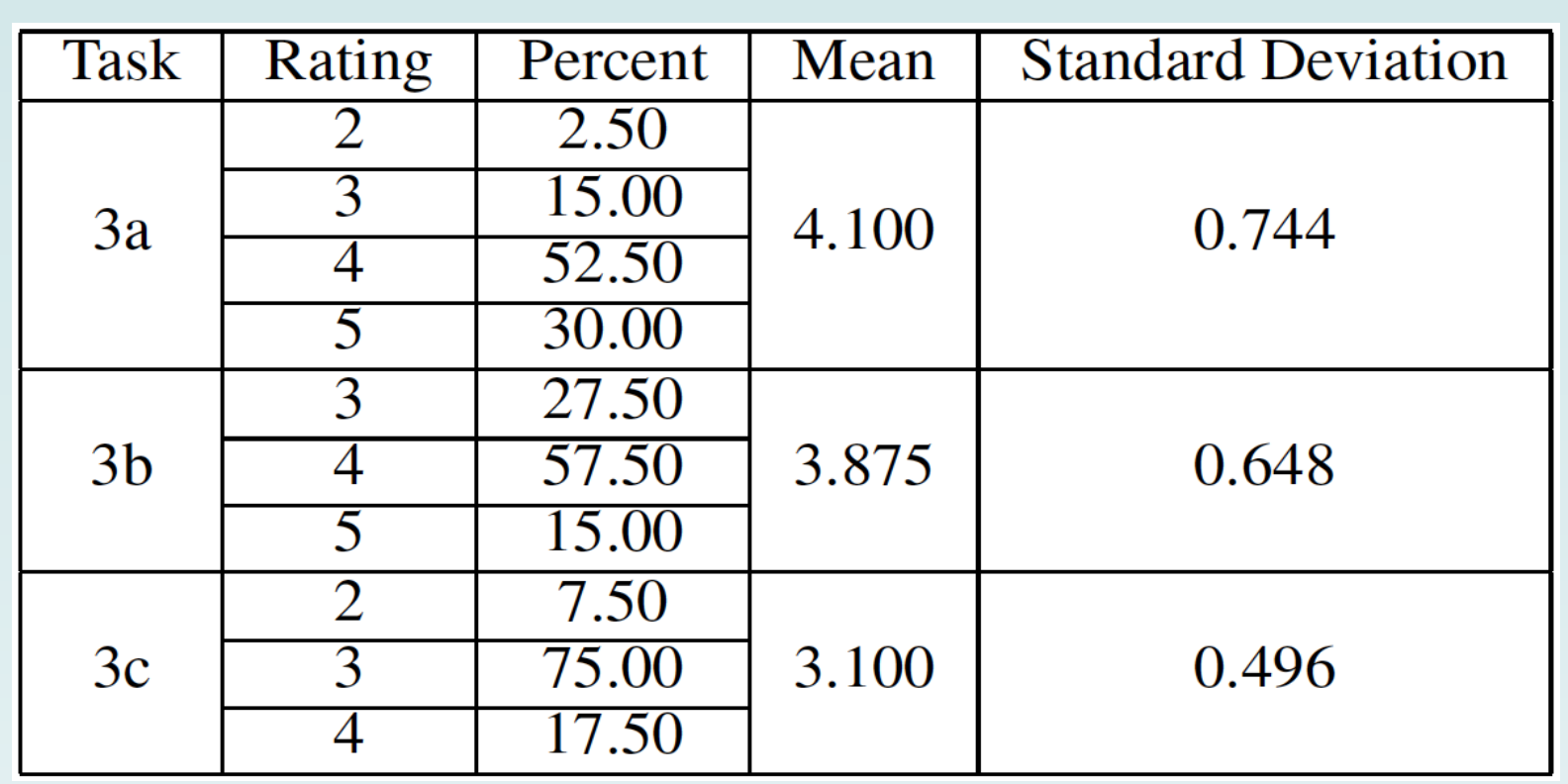

Table 5: Percentage, Mean and Standard Deviation for Replication Task \begin{tabular}{|c|c|c|c|c|}
\hline Rating & Frequency & Percent & Mean & Standard Deviation \\
\hline-3 & 0 & 0.00 & & \\
\hline
\end{tabular} \begin{tabular}{l|l|l|l|l}
-3 & 0 & 0.00 \\
\hline-2 & 0 & 0.00 \\
-1 & 0 & 0
\end{tabular}

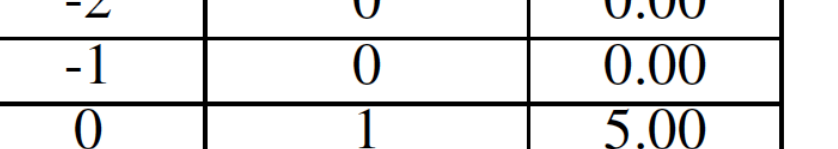
\begin{tabular}{|c|c|c|}
\hline 1 & 8 & 40.00 \\
\hline 2 & 10 & 50.00 \\
\hline
\end{tabular} \begin{tabular}{|c|c|c|}
\hline 3 & 1 & 5.00 \\
\hline Table 7: Overall Satisfaction of User's Output
\end{tabular} \begin{tabular}{l|l|l} 
Tasks & Sketch Tool & Maya \\
\hline
\end{tabular}

\begin{tabular}{|c|c|c|}
\hline Tasks & Sketch Tool & Maya \\
\hline $1 \mathrm{a}$ & $20 \mathrm{~s}$ & 4 mins $28 \mathrm{~s}$ \\
\hline $2 \mathrm{a}$ & $1 \mathrm{~min} 6 \mathrm{~s}$ & $16 \mathrm{mins} 20 \mathrm{~s}$ \\
\hline $3 \mathrm{a}$ & $37 \mathrm{~s}$ & 7 mins $5 \mathrm{~s}$ \\
\hline
\end{tabular}

From Table 5 we can see that most of the subjects can chieve the simple and intermediate tasks. However, the complex iconic gesture task is not performed well.

From Table 6 we can clearly see that our system takes much less time to generate gesture motion compared to Maya.

From Table 7, we can clearly see that about 50 percent of the users are quite satisfied with their overall output. 